\title{
Dose intensity for induction in acute myeloid leukemia: what, when, and for whom?
}

\author{
Shannon R. McCurdy and Selina M. Luger
}

Division of Hematology-Oncology/Department of Medicine, University of Pennsylvania, Philadelphia, PA, USA

Haematologica 2021

Volume 106(10):2544-2554

\section{Correspondence:}

SELINA M. LUGER

selina.luger@pennmedicine.upenn.edu

Received: February 11, 2021.

Accepted: June 11, 2021.

Pre-published: July 29, 2021.

https://doi.org/10.3324/haematol.2020.269134

(C)2021 Ferrata Storti Foundation

Material published in Haematologica is covered by copyright. All rights are reserved to the Ferrata Storti Foundation. Use of published material is allowed under the following terms and conditions:

https://creativecommons.org/licenses/by-nc/4.0/legalcode. Copies of published material are allowed for personal or internal use. Sharing published material for non-commercial purposes is subject to the following conditions:

https://creativecommons. org//icenses/by-nc/4.0/legalcode, sect. 3. Reproducing and sharing published material for commercial purposes is not allowed without permission in writing from the publisher.

\section{ABSTRACT}

Tntensive chemotherapy has been the backbone of the treatment of acute myeloid leukemia (AML) for decades. However, an increase in novel targeted agents, which has been brought about in part by a deeper understanding of the genetic makeup of AML, has led to remission-inducing regimens that do not require traditional cytotoxic agents. Combinations of a hypomethylating agent (HMA) and venetoclax have doubled the chance of remission for patients considered unfit for induction chemotherapy who would have traditionally been offered singleagent HMA. In fact, this regimen may rival the complete remission rate achieved with induction chemotherapy for certain populations such as the very elderly and those with secondary AML, but equivalency has yet to be established. Further advances include the addition of gemtuzumab ozogamicin and FLT3 inhibitors to induction chemotherapy, which improves survival for patients with core-binding factor and FLT3-mutated AML, respectively. Still, much work is needed to improve the outcomes of the highest-risk subgroups: frail patients and those with high-risk cytogenetics and/or TP53 mutations. Promisingly, the landscape of AML therapy is shifting dramatically and no longer is intensity, when feasible, always the best answer for AML.

\section{Introduction}

The initial data for the most widely utilized intensive induction chemotherapy regimen for acute myeloid leukemia (AML), a combination of cytarabine and an anthracycline ("7+3"), were published in 1973. ${ }^{1}$ In the subsequent four decades the question of increasing or decreasing the intensity of induction had been asked multiple times. Various institutions adopted modified cytarabine and anthracycline platforms that included the addition of a third agent such as etoposide and/or employing time sequential therapy. ${ }^{2}$ However, no modifications, other than variations in dose intensities of anthracycline, have been widely adopted. Hypomethylating agents (HMA) and low or intermediate doses of cytarabine have been utilized for patients over 70-75 years old or those considered unfit for intensive therapy due to comorbidity or poor performance status (PS), but intensive induction was the only AML-directed therapy with a known survival benefit. After years of stagnation in new agents, beginning in 2017 the world of AML therapy was uplifted by the approval of new therapies. However, until recently none has provided a true alternative to induction for those individuals who are otherwise induction candidates, and upfront therapy for those who were unfit was still limited. Given the survival benefit seen with venetoclax combination therapies when compared with HMA alone, ${ }^{3}$ the question of intensity has risen again, but with no clear answers regarding whether fit patients would benefit from this intermediate intensity option. Here we will review the literature and provide our position on when to consider dose intensity in the era of novel, less intensive induction strategies.

\section{To treat or not to treat?}

While induction therapy is still considered the standard of care for younger patients with favorable- or intermediate-risk disease, not all patients are deemed 
eligible for standard induction. What has been clearly demonstrated is that AML-directed chemotherapy improves survival for newly diagnosed patients when compared with best supportive care and that this benefit extends to the elderly and those with poor PS. ${ }^{4,5}$ In 1989, Lowenberg and colleagues performed a randomized trial of intensive induction chemotherapy (IC) versus best supportive care combined with mild cytoreductive therapy for relief of progressive symptoms in patients 65 years and older. Despite the concern that older patients would suffer from complications of intensive therapy, survival was significantly better in those treated with IC and frequency of hospitalization was not different between the treatment groups. ${ }^{6}$ Despite these early findings, historically the majority of older adults with AML were not offered chemotherapy. For instance, SEER data for patients with AML aged 65 years and older from 19911996 demonstrated that the median survival was 2.4 months with a dismal 2-year overall survival (OS) of $6 \%$. While those receiving chemotherapy survived longer, only $34 \%$ of patients received chemotherapy. From 2000 to 2009 , treatment rates for older AML patients increased from $35 \%$ to $50 \%$ and leukemia-directed therapy reduced the risk of death by $33 \%$ with a median OS of 18.9 months in those receiving IC, 6.6 months in those receiving HMA, and 1.5 months in patients receiving best supportive care. ${ }^{8}$ In a propensity matched analysis, patients 60 years and older who received IC had a median survival of 197 days whereas those receiving best supportive care had a median survival of 53 days. ${ }^{9}$ Furthermore, a randomized trial comparing IC to best supportive care demonstrated improvement in OS for patients over 65 receiving IC. ${ }^{10}$ The benefit of treatment also appears to extend to patients aged 70-79 years old, in whom a goal of achieving remission has been associated with improved survival. ${ }^{5}$

In one of the original studies of the interaction PS and age in AML, older age and poorer PS had a synergistic effect on early mortality after IC. Thirty-day mortality in patients $\geq 75$ years old with Eastern Cooperative Oncology Group (ECOG) PS of 3 was $82 \%$ compared with $50 \%, 18 \%$, and $14 \%$ for those $\geq 75$ years old with PS of 2,1 , and 0 , respectively. ${ }^{11}$ In AML patients aged 70-79 years old with PS $0-2$ the 8 -week mortality was $8 \%$ in those with intermediate-risk karyotype and $22 \%$ in those with high-risk karyotype, compared with $23 \%$ and $47 \%$ if given palliative therapy only. Interestingly, even in AML patients with an ECOG PS 3-4, 8-week mortality was shown to be $76 \%$ in those treated with palliation and $50 \%$ in those treated with intensive treatment ${ }^{4}$ which suggests that even in those with a poor PS, treatment does not necessarily increase early mortality.

Whether, patients with a poor PS would fare better with less intensive therapy remains to be seen. It is possible that the burden of their disease compromises their PS and that less intensive therapies, which take weeks or months to achieve remission, would not adequately control their leukemia in time to reduce early mortality. For the first time, we have moderate intentsity therapies that offer similar complete resmission (CR) rates compared to IC, albeit with a delay to time of acheiving remission. Studies that compare therapy intensity in patients with poor PS, by fitness level, and by proliferative disease features are needed to determine the best approach in these high-risk patients.

\section{When to treat?}

Time from diagnosis to treatment was historically tied to survival outcomes, with increased mortality associated with delays in initiation of chemotherapy. In 2009, Sekeres et al. published that this was true for patients $<60$ years old, but not for those 60 years and older. ${ }^{12}$ For younger patients, delays beyond 5 days were associated with inferior survival. Importantly, patients with a white blood cell count at diagnosis $>50 \times 10^{9} / \mathrm{L}$ were excluded from the analysis. The authors hypothesized that older AML patients had biologically different disease that was inherently more resistant to chemotherapy. Using chromosomal analysis to identify such patients would allow pursuit of alternatives to standard IC. With further advances in the molecular and chromosomal categorization of AML, delaying therapy to allow individualization of treatment, particularly for older patients, is becoming more common. For instance, our group demonstrated that rapid fluorescence in situ hybridization testing could identify $86 \%$ of patients with myelodysplastic syndrome (MDS)-defining cytogenetics ${ }^{13,14}$ a population that has been shown to benefit from CPX-351 (liposomal daunorubicin and cytarabine). ${ }^{15}$ In addition, rapid identification of core-binding factor (CBF) leukemia through fluorescence in situ hybridization or polymerase chain reaction analysis allows incorporation of gemtuzumab ozogamicin (GO) into induction, which has been shown to improve survival. For all FLT3-mutated patients, the addition of an FLT3 inhibitor to IC is now standard of care. With regard to molecular analyses, the initial management in older patients may change with the presence of an IDH or TP53 mutation. Delaying therapy to allow for genetic classification is, therefore, being increasingly employed.

Given the growing role of chromosomal and molecular testing to tailor initial therapy, Rollig et al. reviewed the outcomes of patients whose treatment was delayed to allow for personalized treatment choices. In their study, with $0-5,6-10,11-15$, and $>15$ days from diagnosis to starting treatment, the 2 -year OS was $51 \%, 48 \%, 44 \%$, and $50 \%$, respectively, with a 30-day mortality rate of approximately $4 \%$ in each group. ${ }^{16}$ Importantly, there was no difference based on whether the initial white blood cell count was $>50 \times 10^{9} / \mathrm{L}$ or $\leq 50 \times 10^{9} / \mathrm{L}$. However, patients with a high white blood cell count, high bone marrow blast count, and/or high lactase dehydrogenase had a shorter time from diagnosis to treatment. ${ }^{16}$ The authors concluded that physicians were appropriately selecting those patients who could postpone therapy initiation and that advances in supportive care such as the use of anti-fungal agents may decrease early mortality to allow safe delays. In addition, stabilizing cytoreductive measures such as hydroxyurea and leukapharesis can be employed to allow informed treatment decisions. As such, we support rapid turnaround of fluorescence in situ hybridization testing for MDS-defining cytogenetics and CBF, as well as molecular analysis for TP53, IDH, and FLT3 mutations. We anticipate that with time, as other genes become targets for leukemia therapy, the list of mutations necessary for rapid testing will grow.

\section{Anthracycline dose}

One of the often debated questions with regard to IC intensity is the ideal dose of anthracycline. In a random- 
ized US intergroup trial, daunorubicin at a dose of 90 $\mathrm{mg} / \mathrm{m}^{2}$ compared with $45 \mathrm{mg} / \mathrm{m}^{2}$ for AML patients younger than 60 years old was associated with a higher CR rate at $70 \%$ versus $57 \%$, improvements in OS at $46.8 \%$ versus $34.6 \%$, and event-free survival at $40.8 \%$ versus $28.4 \%$, respectively. ${ }^{17}$ However, the survival advantage was restricted to patients younger than 50 years old. ${ }^{18}$ With longer follow-up and additional analyses based on molecular classification, it was determined that in patients 50-60 years old who had either an FLT3-internal tandem duplication or an NPM1 mutation higher anthracycline dose was associated with improved OS. ${ }^{19}$ Lowenberg and colleagues demonstrated that in patients aged 60-83 (median, 67) years old who received cytarabine $200 \mathrm{mg} / \mathrm{m}^{2}$ those receiving an escalated dose of daunorubicin to $90 \mathrm{mg} / \mathrm{m}^{2}$ from 45 $\mathrm{mg} / \mathrm{m}^{2}$ had a CR rate of $64 \%$ compared with $54 \%$, but survival endpoints did not differ. ${ }^{20}$ In the subgroup of patients aged $60-65$ years old, the CR rate was $73 \%$ versus $51 \%$, event-free survival was $29 \%$ versus $14 \%$, and OS was $38 \%$ versus $23 \%$, respectively, by anthracycline dose. In the Medical Research Council's (MRC) AML15 randomized phase III trial that compared induction with two cycles of daunorubicin $90 \mathrm{mg} / \mathrm{m}^{2}$ to two cycles of $60 \mathrm{mg} / \mathrm{m}^{2}$, CR rate, toxicity, and OS were similar except for the subgroup of FLT3-mutated patients, in whom the higher dose provided a survival advantage..$^{21,22}$ While toxicity increases with age for escalating anthracycline doses, higher doses should be considered for patients under 60-65 years old, particularly if FLT3-mutated and possibly if NPM1-mutated. Another important consideration is the choice of anthracycline. Idarubicin and daunorubicin have largely been used interchangeably. ${ }^{23-25}$ However, a recent meta-analysis supports the preferential use of idarubicin over daunorubicin, particularly when utilizing a high dose of anthracycline (daunorubicin $90 \mathrm{mg} / \mathrm{m}^{2}$ or equivalent). ${ }^{26}$

\section{Cytarabine dose and CPX-351}

The ideal dosing for cytarabine in combination with an anthracycline for induction is less controversial and appears to be $100-200 \mathrm{mg} / \mathrm{m}^{2} /$ day typically administered as a 24-hour infusion. ${ }^{27}$ Dose escalations beyond 200 $\mathrm{mg} / \mathrm{m}^{2} /$ day have not been associated with further improvements, for instance, increasing the dose to 400 $\mathrm{mg} / \mathrm{m}^{2} /$ day did not improve survival. ${ }^{28}$ Lowenberg and colleagues demonstrated that, when in combination with an anthracycline, high-dose cytarabine $\left(1000 \mathrm{mg} / \mathrm{m}^{2}\right.$ every 12 hours on days 1-5) was associated with no difference in outcomes, but a higher incidence of toxicity, prolonged hospitalization, and delayed hematologic recovery when compared to cytarabine $200 \mathrm{mg} / \mathrm{m}^{2} /$ day as a continuous infusion for 7 days $(7+3) \cdot{ }^{29}$ Early mortality after high-dose cytarabine without an anthracycline was higher than after $7+3$ and is of particular concern in patients with renal dysfunction. Furthermore, the incidence of cerebellar toxicity is much greater with high-dose cytarabine, limiting its use to otherwise fit patients who are in need of a non-anthracycline-containing induction regimen due most often to baseline cardiac dysfunction (ejection fraction $<45 \%{ }^{30}$ ) or cumulative lifetime dose of anthracycline that would exceed doxorubicin $450-550 \mathrm{mg} / \mathrm{m}^{2}$ or equivalent. 30,31

In 2017, CPX-351 (liposomal daunorubicin/cytarabine) was approved for patients with secondary AML as a method to improve the delivery of cytarabine based on a fixed molar ratio. While CPX-351 did not improve survival beyond $7+3$ for all newly diagnosed AML patients, a randomized trial comparing CPX-351 to standard $7+3$ in patients aged $60-75$ years with therapy-related AML, AML from prior $\mathrm{MDS}$, or $\mathrm{AML}$ with MDS-defining cytogenetics demonstrated a significant improvement in median survival of 9.56 versus 5.95 months $(P=0.003)$, 2-year OS of $31.1 \%$ versus $12.3 \%$ and, a higher $C R$ rate of $47.7 \%$ versus $33.3 \%$, $(P=0.016) .{ }^{15}$ However, the consolidation strategy for the control arm utilized cytarabine $100 \mathrm{mg} / \mathrm{m}^{2} /$ day for 5 days and daunorubicin $60 \mathrm{mg} / \mathrm{m}^{2}$ on days 1 and 2 rather than high-dose cytarabine, which is the preferred consolidation regimen and can be utilized either as definitive therapy or as a bridge to hematopoietic cell transplantation (HCT). HMA/venetoclax, discussed more below, competes for the same population of older patients with secondary $\mathrm{AML}$ and produces a promising CR rate, thus we typically consider CPX-351 for patients with secondary AML in whom HMA or HMA/venetoclax has failed.

\section{Triplet chemotherapy}

We have established that chemotherapy intensity is important for treating AML with curative intent, however, until 2017, intensity beyond that established above failed to further improve survival due to improvements in relapse being offset by increased toxic deaths. ${ }^{32-34}$ Etoposide is one of the most widely utilized chemotherapy additions to standard induction, but has not been shown to improve survival when added to $7+3^{35}$ or when utilized as part of time sequential therapy where the $68 \% \mathrm{CR}$ rate and median OS of 17.2 months $^{36}$ are similar to the results associated with conventional $7+3$ on clinical trial..$^{37}$ The addition of cladribine, but not fludarabine, to $7+3$ for AML patients aged 17-60 years was associated with improvement in OS in patients aged 50 to 60 years old, those with a leukocyte count at presentation above $50 \times 10^{9} / \mathrm{L}$, and patients with an unfavorable karyotype. $^{38}$ In contrast, in patients 60-77 years old, the addition of cladribine to $7+3$ did not improve OS or CR. ${ }^{39}$ However, in a subgroup analysis of patients aged 60-65 years old with good- or intermediate-risk cytogenetics there was an OS benefit with the triplet. ${ }^{39}$

Although the addition of a third standard cytotoxic agent to $7+3$ has not been widely adopted, two Food and Drug Administration (FDA)-approved therapies have been shown to boost efficacy in specific populations without significant increases in toxicity: $\mathrm{GO}$ and midostaurin. $\mathrm{GO}$ is a monoclonal antibody to CD33 attached to the traditional cytotoxic drug calicheamicin. Adding $3 \mathrm{mg} / \mathrm{m}^{2}$ of $\mathrm{GO}$ to $7+3$ (daunorubicin $60 \mathrm{mg} / \mathrm{m}^{2}$ ) significantly improved the survival of patients with good-risk AML, ${ }^{40}$ leading to its approval for $\mathrm{CD}_{3} 3^{+} \mathrm{AML}$. A subsequent meta-analysis demonstrated that adding $\mathrm{GO}$ to induction for CBF-AML produced a $20.7 \%$ absolute survival benefit at 6 years. ${ }^{41} \mathrm{GO}$ in combination with $7+3$ has also been explored for NPM1-mutated AML in which it was associated with a reduction in relapse, but a higher early death rate $(10.3 \%$ vs. $5.7 \%)$, which translated into no improvement in event-free survival. ${ }^{42}$ Subgroup analyses suggested a benefit in younger female and FLT3-negative patients, but given that the primary endpoint was not reached, we do not routinely add GO to induction or consolidation for NPM1-mutated AML and instead monitor patients closely for rapid minimal residual disease clearance ${ }^{43}$ and consider 
allogeneic HCT if this is not achieved. While adding GO to induction for patients with intermediate-risk AML was associated with a small improvement in OS, we typically recommend consolidation with HCT in first CR patients. Given the potential for sinusoidal occlusive syndrome after GO and HCT, we reserve GO for patients with CBF-AML, who derive the most benefit ${ }^{44}$ and for whom we do not routinely recommend HCT in first CR. Aside from increasing the risk of sinusoidal occlusive syndrome, $\mathrm{GO}$ also prolongs the duration of cytopenias after $7+3$, but is otherwise well tolerated.

FLT3 (tyrosine kinase domain or internal tandem duplication)-mutated AML is another subtype in which additions to $7+3$ are becoming standard of care. Midostaurin is a protein kinase inhibitor that targets FLT3 and other protein kinases. The phase III Ratify trial demonstrated that patients receiving $7+3$ in combination with midostaurin 50 mg twice daily on days 8-21 compared with 7+3 in combination with placebo had a 4-year OS of $51.4 \%$ versus $44.3 \%$, respectively. ${ }^{45}$ Like GO, midostaurin is also added to consolidation cycles. Midostaurin is well tolerated with rash, nausea, abdominal pain, and diarrhea as the most frequent, but often manageable, side effects. However, in patients over 60 years old receiving midostaurin, cardiac toxicity $(22 \%)$, arrthymias $(10 \%)$, and pulmonary complications $(14 \%)$ were more common than antipicated. ${ }^{46}$ Given the success of midostaurin, the addition of more potent FLT3 inhibitors to induction is being explored in ongoing studies. For instance, $7+3$ plus gilteritinib was associated with a composite CR rate of $93.9 \%$ in early trials. ${ }^{47}$ We anticipate that additional FLT3 inhibitors will be approved for use in combination with induction in the near future. While not yet approved in combination with $7+3$ or $5+2$, the addition of venetoclax to IC is also being explored, with promising early results discussed below.

\section{The argument for intensity in older fit popula- tions}

In a Swedish registry study of older AML patients, early death was dependent on PS and age, but patients administered intensive therapy had a lower early death rate regardless of age..$^{48}$ At the time, HMA use was rare or non-existent $^{49}$ so patients treated with hydroxyurea, lower-dose cytarabine, or best supportive care comprised the majority of the comparator group. ${ }^{49}$ In a study by Bories et al. the OS of patients 60 years and older between 2007-2010 who were selected by their physician to receive IC, or if unfit for IC then azacitidine, or if unfit for azacitidine then best supportive care, was 18.9, 11.3, and 1.8 months, respectively. ${ }^{50}$ One of the limitations of such studies is that PS, disease features, and co-morbidity influence how patients are selected for a given therapy. To adjust for these differences, a propensity score matching analysis that included age, secondary versus de novo AML, bone marrow blast percent, and cytogenetics demonstrated an improvement in OS for IC when compared to azacitidine from 6 months after treatment initiation. ${ }^{50}$ However, PS and co-morbidities were not different between the IC and azacitidine groups and thus not matched. This highlights the subjectivity of fitness; clinicians utilized criteria other than PS and co-morbidity to deem the patient unfit for IC. In a multicenter retrospective analysis of 1,295 patients aged 65 years and older treated between 2008-2012, Sorror and col- leagues tried to account for this type of selection bias by using an AML-composite mode ${ }^{51,52}$ score and found that those who received IC had longer survival than those who received a HMA, including the subset of patients aged 7079 years old. ${ }^{53}$ While this study importantly demonstrates the continued role for IC, the authors acknowledge that it does not include the latest generation of moderate-intensity options. For instance, azacitidine/venetoclax was associated with a composite $\mathrm{CR}$ rate of $66.4 \%$, compared with $28.3 \%$ for single-agent azacitidine, and has the potential to rival the $\mathrm{CR}$ rate of IC in older patients and those with secondary AML.

Another potential advantage of IC is the ability to achieve cure in a small number of patients without need for ongoing HMA therapy or consolidation HCT. For instance, in a study by Heiblig et al. the median OS for AML patients 70 years and older receiving IC, HMA, or best supportive care was 12.4 months, 11.5 months and 2.6 months and the 3 -year OS was $27 \%, 17 \%$, and $6 \%$, respectively. While median OS was similar after IC and HMA, patients receiving HMA were exceedingly unlikely to live beyond 3 years, whereas a very small number of patients receiving IC survived beyond 10 years. ${ }^{54}$ As such, in patients who are not candidates for HCT, upfront intensive options have the potential for long-term survival and may be considered. While some may argue that patients not fit for IC upfront would by definition not be candidates for bone marrow transplantation, OS improvements associated with IC even in poor PS patients contradict this dogma. Furthermore, in older or frail patients, the prolonged stress of HCT may be more difficult to withstand than a single month of intensive treatment.

\section{Clofarabine}

Clofarabine is another moderate-intensity regimen explored in older AML patients who were considered unfit for intensive induction ${ }^{55}$ and at doses of $20 \mathrm{mg} / \mathrm{m}^{2}$ was associated with a CR/CR with incomplete hematologic recovery (CRi) rate of $48 \%$, 30-day mortality of $18 \%,{ }^{56}$ and a median disease-free survival of 37 weeks. ${ }^{55}$ Based on encouraging phase II results, a phase III ECOG ACRIN-led intergroup trial in newly diagnosed AML patients 60 years and older investigated the use of clofarabine $30 \mathrm{mg} / \mathrm{m}^{2}$ for 5 days compared with daunorubicin $60 \mathrm{mg} / \mathrm{m}^{2}$ days $1-3$ and cytarabine $100 \mathrm{mg} / \mathrm{m}^{2}$ days $1-7$ and demonstrated similar $\mathrm{CR}$ rates, but significantly inferior OS for clofarabine-treated patients ( $\mathrm{HR}=1.41)^{57}$ For patients with high-risk cytogenetics there was no difference in OS between the arms. The authors reached the conclusion that $7+3$ induction was still the standard for older fit patients. ${ }^{57}$ Clofarabine in combination with daunorubicin has also been compared to cytarabine and daunorubicin, but with no significant differences in remission or survival outcomes. ${ }^{58}$

\section{The argument for attenuated intensity in older or unfit patients}

Attenuated doses of the combination of anthracycline and cytarabine have not been shown to be of more value in older patients who are fit for induction..$^{59.61}$ However, for patients unfit for IC, lower intensity options have been explored. One of the earliest utilized lower-intensity ther- 
apies, low-dose cytarabine (LDAC), prolongs survival when compared to either hydroxycarbamide or best supportive care. However, the CR rate is only $18 \%$ with a median OS of 18 weeks and there were no responders among patients with high-risk disease. ${ }^{62}$ In an effort to improve upon modest outcomes with LDAC alone, glasdegib, a hedgehog inhibitor that is also being studied in combination with $7+3{ }^{63}$ was combined with LDAC, where it was associated with a CR rate of $17 \%$ and an 8.8 month median survival compared with $2.3 \%$ and 4.9 months for LDAC alone in that study. ${ }^{64}$ Glasdegib is now FDA-approved for use in combination with LDAC and it could be considered in patients with renal failure or those deemed unfit for induction who have already failed HMAbased therapy. However, the widespread adoption of LDAC and glasdegib has been limited by the contemporary approval of LDAC and venetoclax discussed further below.

Azacitidine and decitabine, two HMAs in common use for myeloid diseases, were first approved for MDS in 2004 and 2006, respectively. Despite never receiving FDA approval as single agents for AML, HMAs have been commonly employed to treat unfit older AML patients since that time. The data behind this approach include those from a study of 980 AML patients 70 years and older in whom the median OS was 14.4 months after HMA, 10.8 months after daunorubicin/cytarabine, 5.9 months after LDAC, and 2.1 months with best supportive care. ${ }^{65}$ Propensity score matching showed a significant benefit from HMA. At least two other studies retrospectively compared HMA and IC and suggested that survival was similar. ${ }^{66,67}$ However, when compared to lower-intensity AML chemotherapy such as single-agent GO, LDAC, or best supportive care, nothing outperforms HMA. ${ }^{32,68}$

\section{Venetoclax lower-intensity combination therapies}

In an effort to improve on the modest $\mathrm{CR}$ rate associated with HMA as a single agent, HMA combination therapies, such as lenalidomide/HMA have been explored, ${ }^{69,70}$ but none garnered much attention until venetoclax. Based on encouraging early data, venetoclax/HMA received accelerated approval in 2018 for newly diagnosed AML patients 75 years and older and/or for those with comorbidities that preclude IC..$^{71}$ Full FDA approval followed in 2020 based on the findings of a phase III study in which 431 patients were randomized to either 7 days of azacitidine and 28 days of venetoclax $400 \mathrm{mg}$ daily or azacitidine and placebo. ${ }^{3}$ The OS was 14.7 months in the azacitidine/venetoclax arm and 9.6 months in the azacitidine/placebo arm with a composite CR (CR/CRi) of $66.4 \%$ versus $28.3 \%$, respectively. ${ }^{3}$ By molecular subgroups, CR/CRi was achieved in $75.4 \%$ versus $10.7 \%$ of IDH1/2-mutated patients, $72.4 \%$ versus $36.4 \%$ of FLT3mutated patients, $66.7 \%$ versus $23.5 \%$ of NPM1-mutated patients, and $55.3 \%$ versus $0 \%$ of TP 53 -mutated patients, respectively. The median OS for patients with intermediate-risk cytogenetics treated with azacitidine/venetoclax or azacitidine/placebo, was 20.8 months versus 12.4 months, respectively, whereas it was 7.6 months versus 6.0 months for those with poor-risk cytogenetics (patients with CBF-AML were excluded from the study). Febrile neutropenia occurred in $42 \%$ of patients treated with the combination. Thirty-day mortality was $7 \%$ in the azacitidine/venetoclax group and $6 \%$ in the control group. ${ }^{3}$

Alongside its approval in combination with HMA, venetoclax received accelerated approval in combination with LDAC based on a study of 82 patients aged 63-90 years who were treated with $\mathrm{LDAC}$ at $20 \mathrm{mg} / \mathrm{m}^{2}$ per day on days 1-10 in combination with venetoclax $600 \mathrm{mg}$ daily. ${ }^{72}$ LDAC/venetoclax was associated with a 30-day mortality of $6 \%$, a CR/CRi rate of $44 \%$, and a median OS of 10.1 months. In patients not previously exposed to HMA, the CR/CRi rate was $62 \%$ with a median OS of 13.5 months. ${ }^{72}$ Full approval was granted when subsequent phase III data from patients aged $36-93$ years old demonstrated a $25 \%$ reduction in risk of death in the LDAC/venetoclax versus LDAC/placebo with a CR/CRi rate of $48 \%$ versus $13 \%$, respectively. ${ }^{73}$ Rates of CR/CRi were $72 \%$ in FLT3-mutated, $71 \%$ for $I D H 1 / 2$-mutated, $91 \%$ for NPM1-mutated, and $47 \%$ for TP53-mutated AML. The median survival after LDAC/venetoclax for patients with the corresponding mutations was not reached, 24.4 months, not reached, and 7.2 months. Rates of febrile neutropenia were $32 \%$. These data are particularly promising for AML patients with NPM1 mutations and hint at a high response rate for NPM1-mutated patients treated with cytotoxic chemotherapy and venetoclax, discussed further below.

While not FDA-approved in combination, another lower-intensity option that is being explored is a combination of cytarabine, cladribine, and venetoclax. Phase II data showed a $58 \%$ CR rate with cladribine and LDAC alternating with HMA in older AML patients unfit for intensive induction. ${ }^{74}$ In a subsequent trial, the addition of venetoclax to the prior regimen produced a $94 \%$ CR/CRi rate and is being explored as a moderate-intensity option. $^{75}$

\section{Venetoclax high-intensity combination therapies}

Given its success in combination with lower intensity therapies, venetoclax has also been explored in patients 65 years and older who were fit for intensive chemotherapy. In this trial, venetoclax was given as a 7-day pre-phase ramp up, followed by idarubicin on days 2 and $3(5+2)$, infusional cytarabine on days 2-7, then 7 additional days of venetoclax. Patients in the study were 63-80 years old and had a CR/CRi rate of $72 \%$ ( $97 \%$ in those with de novo AML and $43 \%$ in those with secondary AML). ${ }^{76}$ Interestingly, $a \geq 50 \%$ reduction in blasts after just the prephase venetoclax was documented in patients with NPM1, IDH2, or SRSF2 mutations. A few interesting points can be gleaned from this study: i) that secondary AML likely responds at least as well if not better to $\mathrm{HMA} /$ venetoclax than to $5+2 /$ venetoclax; and ii) a strong signal exists that venetoclax is associated with high response rates in NPM1- and IDH-mutated AML, which might point towards the addition of this agent either to cytotoxic or HMA-based therapy, respectively, to improve outcomes in the future. Venetoclax is also being explored in combination with fludarabine, ara-C, granulocyte colony-stimulating factor, and idarubicin (FLAG-IDA) induction. Among ten newly diagnosed patients treated with this regimen the CR/CRh rate was $91 \%$ with a high rate of minimal residual disease negativitiy." Other intensive combinations such as venetoclax with cladribine, ara$\mathrm{C}$, and idarubicin induction are also showing promise. ${ }^{78}$ 


\section{Disease-related factors}

Primary predictors of response to chemotherapy in AML are molecular and cytogenetic factors and these will be used to guide selection of upfront chemotherapy in the years to come. For instance, in patients 75 years and older treated with IC in the AML Cooperative Group (AMLCG)-1999 trial, the average number of mutations was four and $83 \%$ of patients had a mutation in at least one of the following genes: TET2, DNMT3A, NPM1, SRSF2, ASXL1, TP53, and SF3B1. . While NPM1 and FLT3-internal tandem duplication did not significantly affect OS, adverse-risk cytogenetics (according to the MRC) and IDH1 mutations were associated with poor prognosis and chemorefractoriness. ${ }^{79}$ Overall prognosis was poor with intermediate-risk patients having a 3-year survival rate of $10-15 \%$ and no patients with unfavorablerisk AML being alive at 3 years. ${ }^{79}$ While in this study, NPM1 mutations did not influence survival, an earlier study demonstrated that NPM1 mutations in octogenarians were associated with improved survival after IC ${ }^{80}$ In addition, the CR rate for favorable-risk disease in older patients ranges from $89-95 \%$ for CBF-AML to $69 \%$ for CEBPA-double mutant disease, with CR rates for NPM1mutated AML falling in between. ${ }^{81}$ Furthermore, 3-year OS differs: $47 \%$ versus $21 \%$ for CBF-AML versus CEBPAdouble mutant AML, respectively. ${ }^{81}$ Given the relatively high CR rate, older patients with favorable-risk AML could be considered for intensive induction. In AML patients aged 70-79 years old with PS 0-2, worse cytogenetic risk is associated with higher early mortality, but with IC improving early mortality relative to palliation only. ${ }^{4}$ This supports the pursuit of chemotherapy in patients over 70 years old, but unfortunately does not provide guidance as to the optimal regimen.

In older AML patients with good-risk profiles such as those with NPM1 mutations ${ }^{82}$ or CBF, IC may have the potential to achieve long-term survival or even cure. Early studies demonstrating high response rates of NPM1mutated AML to venetoclax combination ${ }^{73,76}$ suggest that the addition of venetoclax to IC $(7+3$ or $5+2$, etc.) may improve outcomes in the future. On the other hand, older patients with IDH 1 -mutated AML are unlikely to benefit from IC and have excellent response rates to azacitidine/venetoclax. In AML patients with chromosome 5 and/or chromosome 7 abnormalities, ${ }^{, 3}$ MDS-related changes, ${ }^{84}$ and in those with low blast counts, ${ }^{85} \mathrm{HMA}$ therapy has been associated with improved OS when compared with intensive cytarabine-based chemotherapy, supporting HMA-based therapies such as HMA/venetoclax in these populations. Similarly, those with monosomal karyotypes ${ }^{86-89}$ or other adverse cytogenetics who are 75 years and older have essentially $0 \%$ 3-year survival with IC $^{79}$ and should be considered primarily for $\mathrm{HMA} /$ venetoclax. Patients with secondary AML or AML with MDS-defining cytogenetics also do poorly with standard IC and may benefit from initial therapy with azacitidine/venetoclax if they have not previously been treated with HMA. If they are refractory to HMA or HMA/venetoclax, CPX-351 rather than 7+3 induction can be considered, as can the addition of venetoclax to HMA in HMA refractory patients.

TP53-mutated AML is a particular challenge given that it is associated with a 2 -year OS rate of $9 \% .{ }^{90}$ Lower-intensity therapy has been associated with equivalent response rates to those in patients treated with higher-intensity therapy, but with less toxicity. ${ }^{1,92}$ For that reason, IC is less frequently employed in patients with TP53 mutations and clinical trials exploring HMA combinations such as those with venetoclax, magrolimab, or eprenetapopt ${ }^{93}$ are being pursued specifically in this population. Given the poor prognosis of this subgroup to all approved therapies, patients should consider clinical trials if available.

A subgroup of patients who should primarily be considered for IC would be those with highly proliferative AML in whom a delay to CR or effective disease control may lead to excess early mortality. Proliferative leukemia is often associated with CBF, NPM1-, or FLT3-mutated AML, which responds well to initial $7+3$ alone or in combination with a targeted agent and supports the use of IC if a delay to determining molecular and cytogenetic features is not feasible. Given the risk of tumor lysis syndrome with venetoclax, cytoreduction to a white blood cell count $<20 \times 10^{9} / \mathrm{L}$ is preferred prior to initiation and if this is achieved by hydroxyurea then the resulting mucositis may limit oral chemotherapy options and result in missed doses. Thus, if patients are candidates for IC and have proliferative disease, we generally recommend intensive IC.

In addition to age, cytogenetic and molecular risks, and features of proliferative disease, another important consideration when selecting upfront therapy is candidacy for subsequent HCT. The vast majority of older adults have intermediate- or poor-risk cytogenetics, in whom HCT is associated with a survival benefit. Yet, patients may be deemed unfit for HCT at the time of diagnosis because of disease burden affecting PS, but may become candidates for HCT once treated. ${ }^{94}$ However, given that CR is often considered a pre-requisite for HCT, strategies with the highest rates of achieving $C R$ are those that should be pursued. While median survival rates after HMA and IC are similar for patients 60 years and older, the CR rate is significantly lower. ${ }^{67,95-99}$ Given that patients are more likely to undergo HCT if they reach a CR, ${ }^{100}$ then achieving a $\mathrm{CR}$ should be a primary goal if HCT is the destination. However, upfront intensity may not be required if less intensive therapy can achieve a CR without affecting PS. This strategy has been associated with success in early trials of azacitidine/venetoclax in whom some patients went on to HCT. ${ }^{101}$ However, to understand which therapy best achieves $C R$ would require an upfront trial of $7+3$ versus $\mathrm{HMA}$ /venetoclax rather than comparisons of HMA/venetoclax and HMA alone. Thus, there is more work to be done.

\section{Patients' fitness}

One of the primary reasons for a patient not being eligible for IC is being categorized as "unfit." However, lack of a widely utilized consensus definition of what fitness entails often means that this is a subjective clinical assessment. An Italian consensus group provided a definition of unfit that is similar to many criteria used in clinical trial enrollment and includes "(i) age over 75, (ii) congestive heart failure with an ejection fraction $\leq 50 \%$, (iii) DLCO $\leq 65 \%$ or FEV $1 \leq 65 \%$, lung neoplasm, or requiring oxygen, (iv) hemodialysis, or renal cancer and age over 60 , (v) liver cirrhosis Child $\mathrm{B}$ or $\mathrm{C}$ or liver function tests $>3$ times normal values, or age over 60 with biliary or liver cancer or 
viral hepatitis, (vi) resistant infection, (vii) mental illness or cognitive impairment that is uncontrolled, (viii) ECOG PS $\leq 3$ not related to leukemia, (ix) physician judgement." 102 The ninth criteria is critical, as a clinician's gestalt judgment is often a reason that patients are felt to be ineligible for IC. However, the accuracy of this clinician assessment may differ by physician and by practice patterns. In a study by our group, we showed that in newly diagnosed AML patients over 60, a physician's gestalt assessment was less likely to categorize patients as frail and more likely to categorize patients as fit than objective measures of fitness. ${ }^{103}$ Specifically, academic clinicians categorized $37 \%$ of newly diagnosed older AML patients as fit, whereas only 17\% were fit according to Fried's frailty index. ${ }^{103}$

Another key component in determining fitness for IC is PS, which has been tied to early mortality after IC and has a synergistic relationship with age in predicting mortality. ${ }^{11}$ The German cooperative group identified that the risk of early death after intensive chemotherapy was much higher $(24 \%)$ in adults over 60 years old and that the CR rate was much lower (50\%). ${ }^{104}$ Kantarjian et al. evaluated 446 non-CBF AML patients 70 years or older receiving intensive cytarabine-based induction and found that the CR rate was $45 \%$, with an 8 -week mortality of $36 \%$ and a median OS of 4.6 months, with $28 \%$ of patients alive at 1 year. ${ }^{105}$ They identified age $\geq 80$ years, complex karyotype, ECOG PS $2-4$, and creatinine $>1.3 \mathrm{mg} / \mathrm{dL}$ as risk factors for mortality. The authors argued that a $>30 \% 8$ week mortality or a 3 -year survival of $<10 \%$ would prohibit the pursuit of IC. ${ }^{104}$ Even worse, patients with a PS of 3-4 had an 8-week mortality of $77 \%$ and a CR rate of $24 \%$. Although this analysis identified patients at high risk of poor outcomes with IC, there was no comparison to less intensive therapies.

One of the drawbacks to the use of PS at the time of AML diagnosis as a predictor of outcomes is the inability to distinguish impairment due to AML from impairment due to comorbidities. To try to distinguish these factors, Klepin and colleagues used functional status 6 months prior to treatment as recalled by patients and found that it was not predictive of survival, ${ }^{106}$ which supports that the functional status at the moment, regardless of diseaserelated impairment, is relevant. If the AML is highly proliferative and limiting PS, perhaps intensive therapy to achieve a rapid reduction in disease burden is necessary. In fact, this could suggest that poor PS due to disease would be an indication for IC. We know that even in patients with poor PS, IC improves mortality when compared to best supportive care, but whether patients with poor PS would benefit from moderate intensity therapy over IC has yet to be determined.

Geriatric assessments have also been utilized to assess a patient's fitness for IC. For instance, an abridged geriatric assessment was better associated with survival than Karnofsky Performance Scale (KPS) or a physical performance test in older cancer patients ${ }^{107}$ and a 30 -item geriatric assessment for hematologic malignancy performed well in another study. ${ }^{108}$ However, whether these metrics can be utilized to select therapy remains to be seen. In a study by Klepin et al., impaired cognition (defined by a modified mini-mental status examination score $>77$ ) and a short physical performance battery score $<9$ were associated with worse OS and increases in 30-day mortality to $23 \%$ versus $9.6 \%$ in patients 60 years and over receiving treatment with cytarabine-based chemotherapy. ${ }^{106}$ In contrast, in a randomized trial in older patients treated with chemotherapy, geriatric assessment did not influence outcomes. ${ }^{109}$

Comorbidity scores represent another avenue for assessing fitness or potential to support treatment toxicity. As a quick measure of comorbidity, polypharmacy ( $\geq 4$ medications versus $\leq 1$ ) has been associated with 30 -day mortality in older AML patients. ${ }^{110}$ More formal co-morbidity assessments include the hematopoietic cell transplantation- comorbidity index (HCT-CI $)^{111}$ and the Charlson comorbidity index (CCI). ${ }^{112}$ Both of these tools were developed for patients in advanced stages of disease and may overemphasize the frailty of the AML patient at diagnosis. ${ }^{102}$ Nonetheless, in newly diagnosed AML patients 70 years and older receiving IC with $7+3$, CCI was 0 in $68 \%, 1$ in $13 \%$ and 2 in $16 \%$, and patients with a score of $>1$ had an odds ratio of 0.29 of achieving CR $(P=0.05)$. The HCT-CI, which performs well for predicting mortality after HCT, predicted early death after IC for AML in some, ${ }^{113}$ but not in other studies. ${ }^{103,106}$ Sorror and colleagues found that creating a model that added albumin $<3.5 \mathrm{~g} / \mathrm{dL}$, platelet count $<20 \times 10^{9}$ cells $/ \mathrm{L}$, lactate dehydrogenase level, age, and cytogenetic and molecular risk to form an AML composite model outperformed the HCT$\mathrm{CI}$, augmented HCT-CI, and KPS for predicting mortality with AML induction. ${ }^{114}$

One of the limitations of utilizing comorbidity to assess fitness for IC is that some patients may have well-compensated comorbidities that do not strongly influence outcomes whereas other patients may have few comorbidities, but be frail due to lifestyle or genetics. We used Fried's frailty phenotype to predict outcomes in newly diagnosed AML patients 60 years and older and found that $17 \%$ of patients were fit, $33 \%$ were pre-frail, and $50 \%$ were frail. All fit and pre-frail patient survived to 100 days, but the 100-day mortality was $51 \%$ for frail patients $(P=0.01) .{ }^{102}$ In contrast, HCT-CI and clinician's gestalt judgment were not significantly associated with mortality in our study. Interestingly, many of the frail patients who died received HMA-based therapy or best supportive care, whereas all four frail patients who were treated with IC with either $7+3$ or CPX-351 survived. ${ }^{103}$ While this result needs to be validated in a larger population of patients, it is intriguing that fitness (or its inverse, frailty), while predictive of mortality, may not be the ideal way to exclude AML patients from IC.

\section{Summary}

Fortunately, the treatment of AML is evolving rapidly. Ongoing clinical trials are evaluating the role of optimizing intensive therapy with the addition of venetoclax, IDH inhibitors, or FLT3 inhibitors. In addition, the National Institutes of Health and national cooperative groups are developing a study to determine the optimal approach to induction and post-remission therapy using personalized diagnostics including a comprehensive molecular and cytogenetic screening. Based on disease characteristics, risk profile, age and fitness, patients will be assigned to an initial therapy to include evaluating the role of novel therapeutics. Remissions will be assessed for minimal residual disease by flow and subsequent therapy will depend on the depth of initial remission in addition to baseline disease characteristics. 


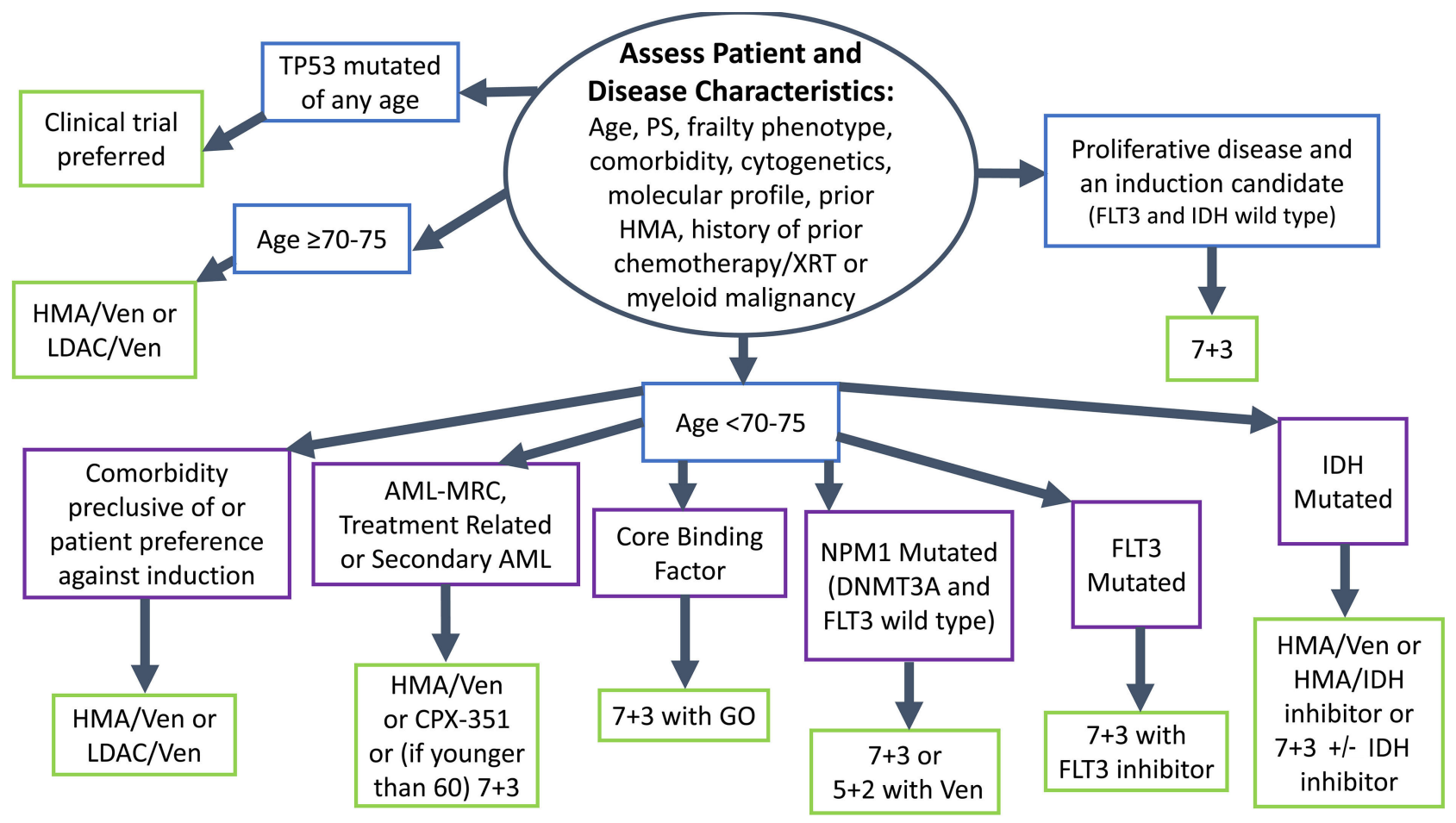

Figure 1. Selection of initial therapy for acute myeloid leukemia. *PS: Performance Status; HMA: hypomethylating agent; XRT: radiation; Ven: venetoclax; LDAC: lowdose ara-C; AML-MRC: acute myeloid leukemia with myelodysplasia-related changes; GO: gemtuzumab ozogamicin.

While looking hopefully to the future, our practice as of now (summarized in Figure 1) is to pursue IC with $7+3$ in patients less than 70 years old with no criteria for exclusion from induction. We add GO to $7+3$ for all patients with CBF-AML, as long as their liver function is adequate, and FLT3 inhibitors to 7+3 from day 8-21 for all FLT3mutated patients. For patients over 70 years old we prefer azacitidine/venetoclax as induction unless they have highly proliferative disease (i.e., extreme hyperleukocytosis) or have cytogenetics or mutations suggestive of chemoresponsiveness (CBF or NPM1). We suspect that a potential third practice-changing triplet could come in the form of venetoclax, cytarabine, and an anthracycline, which has shown early success in NPM1-mutated AML. For patients younger than 70 years old, we would consider azacitidine/venetoclax if they have secondary AML, adverse-risk AML including monosomal karyotype, complex karyotype or MDS-defining cytogenetics. For TP53-mutated
AML patients we recommend a clinical trial if available until the next practice-changing treatment is approved. As leukemia physicians we are fortunate to be working in an era of remarkable progress. First and foremost, we know that the future will yield even more dramatic changes to the treatment of AML than we have been fortunate to witness in the past few years.

\section{Disclosures}

SML has received research support for her institution from Onconova, Kura, Hoffman La Roche, Ariad, and Biosight; and honoraria from Abbvie, Syrox, Jazz, Daiichi-Sankyo, Pfizer, Bristol-Myers Squibb, Acceleron, Agios, and Loxo Oncology. $S R M$ has received research support from Gilead and Aprea.

\section{Contributions}

SML and SRM performed the literature review, evaluated the data, and wrote the manuscript.

\section{References}

1. Yates JW, Wallace HJ Jr, Ellison RR, Holland JF. Cytosine arabinoside (NSC-63878) and daunorubicin (NSC-83142) therapy in acute nonlymphocytic leukemia. Cancer Chemother Rep. 1973;57(4):485-488.

2. Bolanos-Meade J, Karp JE, Guo C, et al. Timed sequential therapy of acute myelogenous leukemia in adults: a phase II study of retinoids in combination with the sequential administration of cytosine arabinoside, idarubicin and etoposide. Leuk Res. 2003;27 (4):313-321.

3. DiNardo CD, Jonas BA, Pullarkat V, et al. Azacitidine and venetoclax in previously untreated acute myeloid leukemia. $\mathrm{N}$ Engl J
Med. 2020;383(7):617-629.

4. Juliusson G, Swedish AML Group. Most 70to 79-year-old patients with acute myeloid leukemia do benefit from intensive treatment. Blood. 2011;117(12):3473-3474.

5. Juliusson G, Billstrom R, Gruber A, et al. Attitude towards remission induction for elderly patients with acute myeloid leukemia influences survival. Leukemia. 2006;20(1):42-47.

6. Lowenberg B, Zittoun R, Kerkhofs H, et al On the value of intensive remission-induction chemotherapy in elderly patients of 65+ years with acute myeloid leukemia: a randomized phase III study of the European Organization for Research and Treatment of Cancer Leukemia Group. J Clin Oncol.
1989.7(9):1268-1274

7. Lang K, Earle CC, Foster T, Dixon D, Van Gool R, Menzin J. Trends in the treatment of acute myeloid leukaemia in the elderly. Drugs Aging. 2005;22(11):943-955.

8. Medeiros BC, Satram-Hoang S, Hurst D, Hoang KQ, Momin F, Reyes C. Big data analysis of treatment patterns and outcomes among elderly acute myeloid leukemia patients in the United States. Ann Hematol. 2015;94(7):1127-1138.

9. Baz R, Rodriguez C, Fu AZ, et al. Impact of remission induction chemotherapy on survival in older adults with acute myeloid leukemia. Cancer. 2007;110(8):1752-1759.

10. Puts MT, Costa-Lima B, Monette J, et al. Medication problems in older, newly diag- 
nosed cancer patients in Canada: how common are they? A prospective pilot study. Drugs Aging. 2009;26(6):519-536

11. Appelbaum FR, Gundacker H, Head DR, et al. Age and acute myeloid leukemia. Blood. 2006;107(9):3481-3485.

12. Sekeres MA, Elson P, Kalaycio ME, et al. Time from diagnosis to treatment initiation predicts survival in younger, but not older, acute myeloid leukemia patients. Blood. 2009;113(1):28-36

13. Nelson ND, McMahon CM, Navarro FE, et al. Rapid fluorescence in situ hybridisation optimises induction therapy for acute myeloid leukaemia. Br J Haematol. 2020;191 (5):935-938.

14. McMahon CM, Nelson N, Ganetsky A, et al. Limited FISH testing for MDS-defining cytogenetic abnormalities rapidly identifies patients with newly diagnosed AML eligible for CPX-351. Blood. 2018;132(Suppl 1):4785.

15. Lancet JE, Uy GL, Cortes JE, et al. CPX-351 (cytarabine and daunorubicin) liposome for injection versus conventional cytarabine plus daunorubicin in older patients with newly diagnosed secondary acute myeloid leukemia. J Clin Oncol. 2018;36(26):26842692.

16. Rollig C, Kramer M, Schliemann C, et al. Does time from diagnosis to treatment affect the prognosis of patients with newly diagnosed acute myeloid leukemia? Blood. 2020;136(7):823-830.

17. Fernandez HF, Sun Z, Yao X, et al. Anthracycline dose intensification in acute myeloid leukemia. N Engl J Med. 2009;361 (13):1249-1259.

18. Lee JH, Joo YD, Kim H, et al. A randomized trial comparing standard versus high-dose daunorubicin induction in patients with acute myeloid leukemia. Blood. 2011;118 (14):3832-3841.

19. Luskin MR, Lee JW, Fernandez HF, et al. Benefit of high-dose daunorubicin in AML induction extends across cytogenetic and molecular groups. Blood. 2016;127(12):15511558.

20. Lowenberg B, Ossenkoppele GJ, van Putten $W$, et al. High-dose daunorubicin in older patients with acute myeloid leukemia. N Engl J Med. 2009;361(13):1235-1248.

21. Burnett AK, Russell NH, Hills RK, et al. A randomized comparison of daunorubicin 90 $\mathrm{mg} / \mathrm{m} 2$ vs $60 \mathrm{mg} / \mathrm{m} 2$ in AML induction: results from the UK NCRI AML17 trial in 1206 patients. Blood. 2015;125(25):38783885.

22. Burnett AK, Russell NH, Hills RK, United Kingdom National Cancer Research Institute Acute Myeloid Leukemia Study Group. Higher daunorubicin exposure benefits FLT3 mutated acute myeloid leukemia. Blood. 2016;128(3):449-452.

23. Berman E, Heller G, Santorsa J, et al. Results of a randomized trial comparing idarubicin and cytosine arabinoside with daunorubicin and cytosine arabinoside in adult patients with newly diagnosed acute myelogenous leukemia. Blood. 1991;77(8):1666-1674.

24. Vogler WR, Velez-Garcia E, Weiner RS, et al. A phase III trial comparing idarubicin and daunorubicin in combination with cytarabine in acute myelogenous leukemia: a Southeastern Cancer Study Group Study. J Clin Oncol. 1992;10(7):1103-1111.

25. Rowe JM, Neuberg D, Friedenberg W, et al. A phase 3 study of three induction regimens and of priming with GM-CSF in older adults with acute myeloid leukemia: a trial by the Eastern Cooperative Oncology Group. Blood. 2004;103(2):479-485.
26. Wang H, Xiao X, Xiao Q, Lu Y, Wu Y. The efficacy and safety of daunorubicin versus idarubicin combined with cytarabine for induction therapy in acute myeloid leukemia: a meta-analysis of randomized clinical trials. Medicine (Baltimore) 2020;99(24):e20094.

27. Rai KR, Holland JF, Glidewell OJ, et al Treatment of acute myelocytic leukemia: a study by Cancer and Leukemia Group B. Blood. 1981;58(6):1203-1212

28. Burnett AK, Milligan D, Goldstone A, et al. The impact of dose escalation and resistance modulation in older patients with acute myeloid leukaemia and high risk myelodysplastic syndrome: the results of the LRF AML14 trial. Br J Haematol. 2009;145(3): 318-332.

29. Lowenberg B, Pabst T, Vellenga E, et al. Cytarabine dose for acute myeloid leukemia. N Engl J Med. 2011;364(11):1027. 1036.

30. Wouters KA, Kremer LC, Miller TL, Herman EH, Lipshultz SE. Protecting against anthracycline-induced myocardial damage: a review of the most promising strategies. $\mathrm{Br} \mathrm{J}$ Haematol. 2005;131(5):561-578.

31. Swain SM, Whaley FS, Ewer MS. Congestive heart failure in patients treated with doxorubicin: a retrospective analysis of three trials. Cancer. 2003:97(11):2869-2879.

32. Burnett AK, Hills RK, Milligan DW, et al. Attempts to optimize induction and consolidation treatment in acute myeloid leukemia: results of the MRC AML12 trial. J Clin Oncol. 2010;28(4):586-595.

33. Goldstone $\mathrm{AH}$, Burnett AK, Wheatley $\mathrm{K}$, et al. Attempts to improve treatment outcomes in acute myeloid leukemia (AML) in older patients: the results of the United Kingdom Medical Research Council AML11 trial. Blood. 2001;98(5):1302-1311.

34. Schaich M, Illmer T, Aulitzky W, et al. Intensified double induction therapy with high dose mitoxantrone, etoposide, mamsacrine and high dose ara-C for elderly acute myeloid leukemia patients aged 61-65 years. Haematologica. 2002;87(8):808-815.

35. Bishop JF, Lowenthal RM, Joshua D, et al. Etoposide in acute nonlymphocytic leukemia. Australian Leukemia Study Group. Blood. 1990;75(1):27-32.

36. Norsworthy KJ, DeZern AE, Tsai HL, et al Timed sequential therapy for acute myel ogenous leukemia: results of a retrospective study of 301 patients and review of the literature. Leuk Res. 2017;61:25-32.

37. Castaigne S, Chevret S, Archimbaud E, et al. Randomized comparison of double induction and timed-sequential induction to a " $3+$ 7" induction in adults with AML: long-term analysis of the Acute Leukemia French Association (ALFA) 9000 study. Blood 2004;104(8):2467-2474.

38. Holowiecki J, Grosicki S, Giebel S, et al. Cladribine, but not fludarabine, added to daunorubicin and cytarabine during induction prolongs survival of patients with acute myeloid leukemia: a multicenter, randomized phase III study. J Clin Oncol. 2012;30(20):2441-2448

39. Pluta A, Robak T, Wrzesien-Kus A, et al. Addition of cladribine to the standard induction treatment improves outcomes in a subset of elderly acute myeloid leukemia patients. Results of a randomized Polish Adult Leukemia Group (PALG) phase II trial. Am J Hematol. 2017;92(4):359-366.

40. Burnett AK, Hills RK, Milligan D, et al. Identification of patients with acute myeloblastic leukemia who benefit from the addition of gemtuzumab ozogamicin results of the MRC AML15 trial. J Clin Oncol. 2011;29(4):369-377.

41. Hills RK, Castaigne S, Appelbaum FR, et al. Addition of gemtuzumab ozogamicin to induction chemotherapy in adult patients with acute myeloid leukaemia: a metaanalysis of individual patient data from randomised controlled trials. Lancet Oncol. 2014;15(9):986-996.

42. Schlenk RF, Paschka P, Krzykalla J, et al Gemtuzumab ozogamicin in NPM1-mutated acute myeloid leukemia: early results from the prospective randomized AMLSC 09-09 phase III study. J Clin Oncol. 2020;38(6):623-632.

43. Ivey A, Hills RK, Simpson MA, et al. Assessment of minimal residual disease in standard-risk AML. N Engl J Med. 2016;374 (5):422-433

44. Mosna F, Papayannidis C, Martinelli G, et al. Complex karyotype, older age, and reduced first-line dose intensity determine poor survival in core binding factor acute myeloid leukemia patients with long-term follow-up. Am J Hematol. 2015;90(6):515-523.

45. Stone RM, Mandrekar SJ, Sanford BL, et al. Midostaurin plus chemotherapy for acute myeloid leukemia with a FLT3 mutation. N Engl J Med. 2017;377(5):454-464.

46. Schlenk RF, Weber D, Fiedler W, et al Midostaurin added to chemotherapy and continued single-agent maintenance therapy in acute myeloid leukemia with FLT3-ITD. Blood. 2019;133(8):840-851

47. Pratz KW, Cherry M, Altman JK, et al. Updated results from a phase 1 study of gilteritinib in combination with induction and consolidation chemotherapy in subjects with newly diagnosed acute myeloid leukemia (AML). Blood. 2018;132(Suppl 1):564.

48. Juliusson G, Hagberg $O$, Lazarevic VL, et al. Improved survival of men 50 to 75 years old with acute myeloid leukemia over a 20-year period. Blood. 2019;134(18):1558-1561.

49. Juliusson G. Older patients with acute myeloid leukemia benefit from intensive chemotherapy: an update from the Swedish Acute Leukemia Registry. Clin Lymphoma Myeloma Leuk. 2011;11(Suppl 1):S54-59.

50. Bories P, Bertoli S, Berard E, et al. Intensive chemotherapy, azacitidine, or supportive care in older acute myeloid leukemia patients: an analysis from a regional healthcare network. Am J Hematol. 2014;89(12): E244-252.

51. Orvain C, Tanguy-Schmidt A, Thepot S, et al. Validation of the revised AML-composite model for the prediction of prognosis in older patients receiving intensive induction therapy. Blood. 2019;134(Suppl 1):1310.

52. Elsayed S, Storer BE, Medeiros BC, et al Augmentation of the acute myeloid leukemia-composite model (AML-CM) with performance status and secondary leukemia. Blood. 2018;132(Supplement 1):3992.

53. Sorror ML, Storer BE, Fathi AT, et al. Multisite 11-year experience of less-intensive versus intensive therapies in acute myeloid leukemia. Blood. 2021;138(5):387-400

54. Heiblig M, Le Jeune C, Elhamri M, et al Treatment patterns and comparative effectiveness in elderly acute myeloid leukemia patients (age 70 years or older): the Lyonuniversity hospital experience. Leuk Lymphoma. 2017;58(1):110-117

55. Kantarjian HM, Erba HP, Claxton D, et al. Phase II study of clofarabine monotherapy in previously untreated older adults with acute myeloid leukemia and unfavorable 
prognostic factors. J Clin Oncol. 2010;28 (4):549-555.

56. Burnett AK, Russell NH, Kell J, et al. European development of clofarabine as treatment for older patients with acute myeloid leukemia considered unsuitable for intensive chemotherapy. J Clin Oncol. 2010;28(14):2389-2395.

57. Foran JM, Sun ZX, Claxton DF, et al. North American Leukemia Intergroup phase III randomized trial of single agent clofarabine as induction and post-remission therapy, and decitabine as maintenance therapy in newly-diagnosed acute myeloid leukemia in older adults (age $>=60$ years): a trial of the ECOG-ACRIN Cancer Research Group (E2906). Blood. 2015;126(23):217.

58. Burnett AK, Russell NH, Hills RK, et al. A comparison of clofarabine with ara-C, each in combination with daunorubicin as induction treatment in older patients with acute myeloid leukaemia. Leukemia. 2017;31(2): 310-317.

59. Bouabdallah R, Lefrere F, Rose C, et al. A phase II trial of induction and consolidation therapy of acute myeloid leukemia with weekly oral idarubicin alone in poor risk elderly patients. Leukemia. 1999;13(10):14911496.

60. Huang BT, Zhao WH, Zeng QC, Li BS, Chen RL. Standard intensive chemotherapy is less effective and far more toxic than attenuated induction and post-induction regimen in elderly patients with acute myeloid leukemia. Med Oncol. 2014;31(5):962.

61. Liu H, Fu R, Li L, et al. Comparison of reduced-intensity idarubicin and daunorubicin plus cytarabine as induction chemotherapy for elderly patients with newly diagnosed acute myeloid leukemia. Clin Drug Investig. 2017;37(2):167-174.

62. Burnett AK, Milligan D, Prentice AG, et al. A comparison of low-dose cytarabine and hydroxyurea with or without all-trans retinoic acid for acute myeloid leukemia and high-risk myelodysplastic syndrome in patients not considered fit for intensive treatment. Cancer. 2007;109(6):1114-1124.

63. Savona MR, Pollyea DA, Stock W, et al. Phase Ib study of glasdegib, a hedgehog pathway inhibitor, in combination with standard chemotherapy in patients with AML or high-risk MDS. Clin Cancer Res. 2018;24(10):2294-2303

64. Cortes JE, Heidel FH, Hellmann A, et al. Randomized comparison of low dose cytarabine with or without glasdegib in patients with newly diagnosed acute myeloid leukemia or high-risk myelodysplastic syndrome. Leukemia. 2019;33(2): 379-389.

65. Talati C, Goldberg AD, Przespolewski A, et al. Comparison of induction strategies and responses for acute myeloid leukemia patients after resistance to hypomethylating agents for antecedent myeloid malignancy. Leuk Res. 2020;93:106367

66. Dombret H, Seymour JF, Butrym A, et al. International phase 3 study of azacitidine vs conventional care regimens in older patients with newly diagnosed AML with $>30 \%$ blasts. Blood. 2015;126(3):291-299.

67. Quintas-Cardama A, Ravandi F, Liu-Dumlao $\mathrm{T}$, et al. Epigenetic therapy is associated with similar survival compared with intensive chemotherapy in older patients with newly diagnosed acute myeloid leukemia. Blood. 2012;120(24):4840-4845

68. Kantarjian HM, Thomas XG, Dmoszynska A, et al. Multicenter, randomized, openlabel, phase III trial of decitabine versus patient choice, with physician advice, of either supportive care or low-dose cytarabine for the treatment of older patients with newly diagnosed acute myeloid leukemia. J Clin Oncol. 2012;30(21):2670-2677.

69. Visani G, Ferrara F, Di Raimondo F, et al. Low-dose lenalidomide plus cytarabine induce complete remission that can be predicted by genetic profiling in elderly acute myeloid leukemia patients. Leukemia. 2014;28(4):967-970

70. Visani G, Ferrar F, Di Raimondo F, et al. Lowdose lenalidomide plus cytarabine in very elderly, unfit acute myeloid leukemia patients: final result of a phase II study. Leukemia Res. 2017;62:77-83.

71. DiNardo CD, Pratz K, Pullarkat V, et al. Venetoclax combined with decitabine or azacitidine in treatment-naive, elderly patients with acute myeloid leukemia. Blood. 2019;133(1):7-17.

72. Wei AH, Strickland SA Jr, Hou JZ, et al. Venetoclax combined with low-dose cytarabine for previously untreated patients with acute myeloid leukemia: results from a phase Ib/II study. J Clin Oncol. 2019;37(15): 1277-1284.

73. Wei AH, Montesinos P, Ivanov V, et al. Venetoclax plus LDAC for newly diagnosed AML ineligible for intensive chemotherapy: a phase 3 randomized placebo-controlled trial. Blood. 2020;135(24):2137-2145.

74. Kadia TM, Cortes J, Ravandi F, et al. Cladribine and low-dose cytarabine alternating with decitabine as front-line therapy for elderly patients with acute myeloid leukaemia: a phase 2 single-arm trial. Lancet Haematol. 2018;5(9):e411-e421.

75. Kadia TM, Borthakur G, Pemmaraju N, et al. Phase II study of venetoclax added to cladribine plus low dose AraC (LDAC) alternating with 5 -azacytidine demonstrates high rates of minimal residual disease (MRD) negative complete remissions (CR) and excellent tolerability in older patients with newly diagnosed acute myeloid leukemia (AML). Blood. 2020;136(Suppl 1):17-19.

76. Chua CC, Roberts AW, Reynolds J, et al. Chemotherapy and Venetoclax in Elderly Acute Myeloid Leukemia Trial (CAVEAT): a phase Ib dose-escalation study of venetoclax combined with modified intensive chemotherapy. J Clin Oncol. 2020;38(30): 3506-3517.

77. Aboudalle I, Konopleva MY, Kadia TM, et al. A phase Ib/II study of the BCL-2 inhibitor venetoclax in combination with standard intensive AML induction/consolidation therapy with FLAG-IDA in patients with newly diagnosed or relapsed/refractory AML. Blood. 2019;134(Suppl 1):176.

78. Kadia TM, Garcia-Manero G, Yilmaz M, et al. Venetoclax (Ven) added to intensive chemo with cladribine, idarubicin, and $\mathrm{AraC}$ (CLIA) achieves high rates of durable complete remission with low rates of measurable residual disease (MRD) in pts with newly diagnosed acute myeloid leukemia (AML). J Clin Oncol. 2020;38(15-suppl): 7539.

79. Prassek VV, Rothenberg-Thurley $M$, Sauerland MC, et al. Genetics of acute myeloid leukemia in the elderly: mutation spectrum and clinical impact in intensively treated patients aged 75 years or older Haematologica. 2018;103(11):1853-1861.

80. Wetzler M, Mrozek K, Kohlschmidt J, et al. Intensive induction is effective in selected octogenarian acute myeloid leukemia patients: prognostic significance of karyotype and selected molecular markers used in the European LeukemiaNet classification.
Haematologica. 2014:99(2):308-313.

81. Mrozek K, Marcucci G, Nicolet D, et al. Prognostic significance of the European LeukemiaNet standardized system for reporting cytogenetic and molecular alterations in adults with acute myeloid leukemia. J Clin Oncol. 2012;30(36):4515-4523.

82. Daver N, Liu Dumlao T, Ravandi F, et al. Effect of NPM1 and FLT3 mutations on the outcomes of elderly patients with acute myeloid leukemia receiving standard chemotherapy. Clin Lymphoma Myeloma Leuk. 2013;13(4):435-440.

83. Ravandi F, Issa JP, Garcia-Manero G, et al. Superior outcome with hypomethylating therapy in patients with acute myeloid leukemia and high-risk myelodysplastic syndrome and chromosome 5 and 7 abnormalities. Cancer. 2009;115(24):5746-5751.

84. Seymour JF, Dohner H, Butrym A, et al. Azacitidine improves clinical outcomes in older patients with acute myeloid leukaemia with myelodysplasia-related changes compared with conventional care regimens. BMC Cancer. 2017:17(1):852

85. Fenaux P, Mufti GJ, Hellstrom-Lindberg E, et al. Azacitidine prolongs overall survival compared with conventional care regimens in elderly patients with low bone marrow blast count acute myeloid leukemia. J Clin Oncol. 2010;28(4):562-569.

86. Breems DA, Lowenberg B. Acute myeloid leukemia with monosomal karyotype at the far end of the unfavorable prognostic spectrum. Haematologica. 2011;96(4):491-493.

87. Becker H, Pfeifer D, Ihorst G, et al Monosomal karyotype and chromosome $17 p$ loss or TP53 mutations in decitabinetreated patients with acute myeloid leukemia. Ann Hematol. 2020;99(7):15511560

88. Breems DA, Van Putten WL, Lowenberg B. The impact of abn(17p) and monosomy $5 / \operatorname{del}(5 q)$ on the prognostic value of the monosomal karyotype in acute myeloid leukemia. Blood. 2013;121(15):3056-3057.

89. Breems DA, Van Putten WL, De Greef GE, et al. Monosomal karyotype in acute myeloid leukemia: a better indicator of poor prognosis than a complex karyotype. J Clin Oncol. 2008;26(29):4791-4797.

90. Kadia TM, Jain P, Ravandi F, et al. TP53 mutations in newly diagnosed acute myeloid leukemia: clinicomolecular characteristics, response to therapy, and outcomes. Cancer. 2016;122(22):3484-3491.

91. Rucker FG, Schlenk RF, Bullinger L, et al. TP53 alterations in acute myeloid leukemia with complex karyotype correlate with specific copy number alterations, monosomal karyotype, and dismal outcome. Blood. 2012;119(9):2114-2121.

92. Boddu P, Kantarjian H, Ravandi F, et al. Outcomes with lower intensity therapy in TP53-mutated acute myeloid leukemia. Leuk Lymphoma. 2018;59(9):2238-2241

93. Cluzeau T, Sebert M, Rahmé R, et al. Eprenetapopt plus azacitidine in TP53mutated myelodysplastic syndromes and acute myeloid leukemia: a phase II study by the Groupe Francophone des Myélodysplasies (GFM). J Clin Oncol 2021:39(14):1575-1583.

94. Prebet T, Gore SD, Esterni B, et al. Outcome of high-risk myelodysplastic syndrome after azacitidine treatment failure. J Clin Oncol. 2011;29(24):3322-3327.

95. Gupta N, Miller A, Gandhi S, et al Comparison of epigenetic versus standard induction chemotherapy for newly diagnosed acute myeloid leukemia patient 
$>/=60$ years old. Am J Hematol. 2015;90(7): 639-646.

96. Oh SB, Park SW, Chung JS, et al. Therapeutic decision-making in elderly patients with acute myeloid leukemia: conventional intensive chemotherapy versus hypomethylating agent therapy. Ann Hematol. 2017;96(11):1801-1809.

97. van der Helm LH, Scheepers ER, Veeger NJ, et al. Azacitidine might be beneficial in a subgroup of older AML patients compared to intensive chemotherapy: a single centre retrospective study of 227 consecutive patients. J Hematol Oncol. 2013;6:29.

98. van der Helm LH, Veeger NJ, Kooy M, et al. Azacitidine results in comparable outcome in newly diagnosed AML patients with more or less than $30 \%$ bone marrow blasts. Leuk Res. 2013;37(8):877-882.

99. Lao Z, Yiu R, Wong GC, Ho A. Treatment of elderly patients with acute myeloid leukemia with azacitidine results in fewer hospitalization days and infective complications but similar survival compared with intensive chemotherapy. Asia Pac J Clin Oncol. 2015;11(1):54-61.

100. Choi EJ, Lee JH, Park HS, et al. Decitabine versus intensive chemotherapy for elderly patients with newly diagnosed acute myeloid leukemia. Clin Lymphoma Myeloma Leuk. 2019;19(5):290-299.

101.Lubbert M, Bertz H, Muller MJ, Finke J. When azanucleoside treatment can be curative: nonintensive bridging strategy before allografting in older patients with myelodysplastic syndrome/acute myeloid leukemia. J Clin Oncol. 2013;31(6):822-823.
102. Ferrara F Barosi G Venditti A, et al. Consensus-based definition of unfitness to intensive and non-intensive chemotherapy in acute myeloid leukemia: a project of SIE, SIES and GITMO group on a new tool for therapy decision making. Leukemia. 2013; 27(5):997-999.

103. McCurdy SR, Gier SH, Babushok DV, et al. Fried frailty phenotype predicts mortality for newly diagnosed older patients with acute myeloid leukemia or high risk myelodysplastic syndrome. Blood. 2019;134 (Suppl 1):2209.

104. Krug U, Rollig C, Koschmieder A, et al. Complete remission and early death after intensive chemotherapy in patients aged 60 years or older with acute myeloid leukaemia: a web-based application for prediction of outcomes. Lancet. 2010;376 (9757):2000-2008.

105. Kantarjian H, Ravandi F, O'Brien S, et al. Intensive chemotherapy does not benefit most older patients (age 70 years or older) with acute myeloid leukemia. Blood. 2010;116(22):4422-4429.

106. Klepin HD, Geiger AM, Tooze JA, et al. Geriatric assessment predicts survival for older adults receiving induction chemotherapy for acute myelogenous leukemia. Blood. 2013;121(21):4287-4294.

107. Ghosn M, Ibrahim T, El Rassy E, Nassani N, Ghanem S, Assi T. Abridged geriatric assessment is a better predictor of overall survival than the Karnofsky Performance Scale and Physical Performance Test in elderly patients with cancer. J Geriatr Oncol. 2017;8(2):128132.
108. Bonanad S, De la Rubia J, Gironella M, et al Development and psychometric validation of a brief comprehensive health status assessment scale in older patients with hematological malignancies: the GAH Scale. J Geriatr Oncol. 2015;6(5):353-361.

109. Magnuson A, Lemelman T, Pandya C, et al Geriatric assessment with management intervention in older adults with cancer: a randomized pilot study. Support Care Cancer. 2018;26(2):605-613.

110. Elliot K, Tooze JA, Geller R, et al. The prognostic importance of polypharmacy in older adults treated for acute myelogenous leukemia (AML). Leuk Res. 2014;38(10): 1184-1190.

111. Sorror ML, Maris MB, Storb R, et al Hematopoietic cell transplantation (HCT) specific comorbidity index: a new tool for risk assessment before allogeneic HCT. Blood. 2005;106(8):2912-2919.

112. Charlson ME, Pompei P, Ales KL MacKenzie CR. A new method of classifying prognostic comorbidity in longitudinal studies: development and validation. J Chronic Dis. 1987;40(5):373-383.

113. Etienne A, Esterni B, Charbonnier A, et al Comorbidity is an independent predictor of complete remission in elderly patients receiving induction chemotherapy for acute myeloid leukemia. Cancer. 2007;109(7): 1376-1383.

114. Sorror ML, Storer BE, Fathi AT, et al Development and validation of a novel acute myeloid leukemia-composite model to estimate risks of mortality. JAMA Oncol. 2017;3(12):1675-1682 